ELECTRONIC RESEARCH ANNOUNCEMENTS OF THE AMERICAN MATHEMATICAL SOCIETY

Volume 9, Pages 19-25 (February 3, 2003)

S $1079-6762(03) 00108-2$

\title{
A $C^{0}$-THEORY FOR THE BLOW-UP OF SECOND ORDER ELLIPTIC EQUATIONS OF CRITICAL SOBOLEV GROWTH
}

\author{
OLIVIER DRUET, EMMANUEL HEBEY, AND FRÉDÉRIC ROBERT \\ (Communicated by Tobias Colding)
}

\begin{abstract}
Let $(M, g)$ be a smooth compact Riemannian manifold of dimension $n \geq 3$, and $\Delta_{g}=-\operatorname{div}_{g} \nabla$ the Laplace-Beltrami operator. Also let $2^{\star}$ be the critical Sobolev exponent for the embedding of the Sobolev space $H_{1}^{2}(M)$ into Lebesgue spaces, and $h$ a smooth function on $M$. Elliptic equations of critical Sobolev growth like

$$
\Delta_{g} u+h u=u^{2^{\star}-1}
$$

have been the target of investigation for decades. A very nice $H_{1}^{2}$-theory for the asymptotic behaviour of solutions of such an equation is available since the 1980 's. In this announcement we present the $C^{0}$-theory we have recently developed. Such a theory provides sharp pointwise estimates for the asymptotic behaviour of solutions of the above equation.
\end{abstract}

Let $(M, g)$ be a smooth compact Riemannian manifold of dimension $n \geq 3$. We denote by $H_{1}^{2}(M)$ the standard Sobolev space of functions in $L^{2}$ with one derivative in $L^{2}$. We consider equations like

$$
\Delta_{g} u+h u=u^{2^{\star}-1},
$$

where $\Delta_{g}=-\operatorname{div}_{g} \nabla$ is the Laplace-Beltrami operator, $2^{\star}=2 n /(n-2)$ is the critical Sobolev exponent for the embedding of the Sobolev space $H_{1}^{2}(M)$ into Lebesgue spaces, $h$ is a $C^{0, \theta}$ function on $M, 0<\theta<1$, and $u$ is required to be positive. We regard (1) as a possible model for second order elliptic equations of critical Sobolev growth. We let $\left(h_{\alpha}\right)$ be a sequence of $C^{0, \theta}$ functions on $M, 0<\theta<1$, and let $\left(u_{\alpha}\right)$ be a bounded sequence in $H_{1}^{2}(M)$ of solutions of (1) in the sense that for any $\alpha$,

$$
\Delta_{g} u_{\alpha}+h_{\alpha} u_{\alpha}=u_{\alpha}^{2^{\star}-1}
$$

and $\left\|u_{\alpha}\right\|_{H_{1}^{2}} \leq \Lambda$, where $\Lambda>0$ is independent of $\alpha$. We also assume that the $h_{\alpha}$ 's are uniformly bounded and that they converge in $L^{2}$ to some limiting function $h_{\infty}$. Then, thanks to Struwe [22], we know how to describe the asymptotic behaviour of the $u_{\alpha}$ 's as $\alpha \rightarrow+\infty$. More precisely, it follows from Struwe [22] that, up to a subsequence,

$$
u_{\alpha}=u^{0}+\sum B_{\alpha}^{i}+R_{\alpha}
$$

Received by the editors November 4, 2002 and, in revised form, December 16, 2002.

2000 Mathematics Subject Classification. Primary 35J60; Secondary 58J05.

Key words and phrases. Critical elliptic equations, blow-up behaviour, bubbles. 
where $u^{0}$ is a solution of the limit equation

$$
\Delta_{g} u+h_{\infty} u=u^{2^{\star}-1},
$$

the sum on the right hand side of (3) is a finite sum over $i, B_{\alpha}^{i}$ is a bubble obtained by rescaling fundamental positive solutions of the Euclidean equation $\Delta u=u^{2^{\star}-1}$, and the $R_{\alpha}$ 's are lower order terms which converge strongly to 0 in $H_{1}^{2}(M)$. This asymptotic description provides a very satisfactory $H_{1}^{2}$-theory for the asymptotic behaviour of solutions of equations like (11). Let us assume now that the $h_{\alpha}$ 's converge $C^{0, \theta}$ to $h_{\infty}$ for some $0<\theta<1$. An important issue in the study of equations like (1D) is to get a theory in which the above asymptotic description holds also in the $C^{0}$-space, where pointwise estimates are involved. Such a $C^{0}$ theory was developed in Druet, Hebey and Robert [9]. We present the theory in this announcement.

We know from the Euclidean Sobolev inequality that there exists $K>0$ such that for any smooth function $u$ with compact support in $\mathbb{R}^{n}$,

$$
\left(\int_{\mathbb{R}^{n}}|u|^{2^{\star}} d x\right)^{1 / 2^{\star}} \leq K\left(\int_{\mathbb{R}^{n}}|\nabla u|^{2} d x\right)^{1 / 2} .
$$

The sharp constant $K$ in this inequality is

$$
K_{n}=\sqrt{\frac{4}{n(n-2) \omega_{n}^{2 / n}}},
$$

where $\omega_{n}$ is the volume of the unit $n$-sphere. We let $u$ be the function on $\mathbb{R}^{n}$ given by

$$
u(x)=\left(1+\frac{|x|^{2}}{n(n-2)}\right)^{1-\frac{n}{2}} .
$$

It is easily seen that $u$ is an extremal function for the sharp Euclidean Sobolev inequality. Thanks to Caffarelli, Gidas and Spruck [3, $u$ is also the unique positive solution of the critical Euclidean equation

$$
\Delta u=u^{2^{\star}-1}
$$

which is such that $u(0)=\max _{\mathbb{R}^{n}} u=1$. Its energy $E(u)=\|u\|_{2^{\star}}$ is given by $E(u)=K_{n}^{-(n-2) / 2}$. From now on, we let $(M, g)$ be a smooth compact Riemannian manifold of dimension $n \geq 3$. We also let $\left(x_{\alpha}\right)$ be a converging sequence of points in $M$, and $\left(\mu_{\alpha}\right)$ a sequence of positive real numbers converging to 0 as $\alpha \rightarrow+\infty$. We define the standard bubble with respect to the $x_{\alpha}$ 's and $\mu_{\alpha}$ 's as the sequence $\left(B_{\alpha}\right)$ of functions on $M$ given by

$$
B_{\alpha}(x)=\left(\frac{\mu_{\alpha}}{\mu_{\alpha}^{2}+\frac{d_{g}\left(x_{\alpha}, x\right)^{2}}{n(n-2)}}\right)^{\frac{n-2}{2}},
$$

where $d_{g}$ is the distance with respect to $g$. In other words, standard bubbles are rescalings of fundamental solutions of the critical Euclidean equation $\Delta u=u^{2^{\star}-1}$. Now we return to equation (2), and we assume that there exist $0<\theta<1$ and a $C^{0, \theta}$ function $h_{\infty}$ on $M$ such that

$$
\begin{aligned}
& \text { the operator } \Delta_{g}+h_{\infty} \text { is coercive, and } \\
& h_{\alpha} \rightarrow h_{\infty} \text { in } C^{0, \theta}(M) \text { as } \alpha \rightarrow+\infty .
\end{aligned}
$$


We let $\left(u_{\alpha}\right)$ be a sequence of solutions of (2), $u_{\alpha} \in C^{2, \theta}(M), u_{\alpha}$ positive. The existence of $u_{\alpha}$ implies the coercivity of the operator $\Delta_{g}+h_{\alpha}$. An estimate like the one in Theorem 1 below implies the coercivity of the operator $\Delta_{g}+h_{\infty}$ when $N \geq 1$. We also assume that there exists $\Lambda>0$ such that $E\left(u_{\alpha}\right) \leq \Lambda$ for all $\alpha$, where $E(u)=\|u\|_{2^{\star}}$. Multiplying (2) by $u_{\alpha}$ and integrating over $M$, it is easily seen that the $u_{\alpha}$ 's are bounded in $H_{1}^{2}(M)$. Up to a subsequence, we may therefore assume that for some $u^{0} \in H_{1}^{2}(M)$,

$$
u_{\alpha} \rightarrow u^{0} \text { weakly in } H_{1}^{2}(M)
$$

as $\alpha \rightarrow+\infty$. Then $u^{0}$ is a solution of the limit equation

$$
\Delta_{g} u+h_{\infty} u=u^{2^{\star}-1} .
$$

By the maximum principle and regularity theory, $u^{0} \in C^{2, \theta}(M)$ and either $u^{0} \equiv 0$ or $u^{0}>0$ everywhere in $M$. As already mentioned, we know from Struwe 22 that the $u_{\alpha}$ 's express as $u^{0}$, plus a sum of standard bubbles, plus lower order terms in $H_{1}^{2}(M)$. We want to prove that the $u_{\alpha}$ 's are $C^{0}$-controlled, from below and from above, by $u^{0}$ and standard bubbles. Our theorem is as follows:

Theorem 1. Let $(M, g)$ be a smooth compact Riemannian manifold of dimension $n \geq 3,\left(h_{\alpha}\right)$ a sequence of $C^{0, \theta}$ functions on $M$ such that (4) is satisfied, and $\left(u_{\alpha}\right)$ a sequence of positive solutions to (2) such that $E\left(u_{\alpha}\right) \leq \Lambda$ for some $\Lambda>0$ and all $\alpha$. Then there exist $N \in \mathbb{N}$, converging sequences $\left(x_{i, \alpha}\right)$ in $M$, and sequences $\left(\mu_{i, \alpha}\right)$ of positive real numbers converging to $0, i=1, \ldots, N$, such that, up to a subsequence,

$$
\begin{aligned}
& \left(1-\varepsilon_{\alpha}\right) u^{0}(x)+\frac{1}{C} \sum_{i=1}^{N}\left(\frac{\mu_{i, \alpha}}{\mu_{i, \alpha}^{2}+\frac{d_{g}\left(x_{i, \alpha}, x\right)^{2}}{n(n-2)}}\right)^{\frac{n-2}{2}} \\
& \quad \leq u_{\alpha}(x) \leq\left(1+\varepsilon_{\alpha}\right) u^{0}(x)+C \sum_{i=1}^{N}\left(\frac{\mu_{i, \alpha}}{\mu_{i, \alpha}^{2}+\frac{d_{g}\left(x_{i, \alpha}, x\right)^{2}}{n(n-2)}}\right)^{\frac{n-2}{2}}
\end{aligned}
$$

for all $x \in M$ and all $\alpha$, where $u^{0}$ is the solution of the limit equation (6) given by (5), $C>1$ is independent of $\alpha$ and $x$, and $\left(\varepsilon_{\alpha}\right)$, independent of $x$, is a sequence of positive real numbers converging to 0 as $\alpha \rightarrow+\infty$. In particular, the $u_{\alpha}$ 's are $C^{0}$-controlled, on both sides, by $u^{0}$ and standard bubbles.

A complement to Theorem 1 is that $C$ can be chosen as close as we want to 1 if we restrict the equation in Theorem 1 to small neighbourhoods of the geometrical blow-up points, defined as the limits of the $x_{i, \alpha}$ 's. For instance, if $u^{0} \neq \equiv 0$, or if the $u_{\alpha}$ 's just have one geometrical blow-up point, then for any $\varepsilon>0$, there exists $\delta_{\varepsilon}>0$ such that, up to a subsequence,

$$
\begin{aligned}
& \left(1-\varepsilon_{\alpha}\right) u^{0}(x)+\frac{1}{1+\varepsilon} \sum_{i=1}^{N}\left(\frac{\mu_{i, \alpha}}{\mu_{i, \alpha}^{2}+\frac{d_{g}\left(x_{i, \alpha}, x\right)^{2}}{n(n-2)}}\right)^{\frac{n-2}{2}} \\
& \quad \leq u_{\alpha}(x) \leq\left(1+\varepsilon_{\alpha}\right) u^{0}(x)+(1+\varepsilon) \sum_{i=1}^{N}\left(\frac{\mu_{i, \alpha}}{\mu_{i, \alpha}^{2}+\frac{d_{g}\left(x_{i, \alpha}, x\right)^{2}}{n(n-2)}}\right)^{\frac{n-2}{2}}
\end{aligned}
$$

for all $\alpha$, all $x_{0} \in \mathcal{S}$, and all $x \in B_{x_{0}}\left(\delta_{\varepsilon}\right)$, where $\mathcal{S}$ is the set consisting of the limits of the $x_{i, \alpha}$ 's as $\alpha \rightarrow+\infty$, and $B_{x_{0}}\left(\delta_{\varepsilon}\right)$ is the geodesic ball of center $x_{0}$ and radius 
$\delta_{\varepsilon}$. Outside the $B_{x}\left(\delta_{\varepsilon}\right)^{\text {'s }}, x \in \mathcal{S}$, the $u_{\alpha}$ 's converge $C^{2, \theta}$ to $u^{0}$. The estimate then extends to $M$ in the particular case where $u^{0} \not \equiv 0$. A refined estimate on the $u_{\alpha}$ 's is given below in (77). Another complement to Theorem 11 is that the bubbles in this theorem satisfy the Struwe $H_{1}^{2}$-decomposition [22]. More precisely, we also have that for any $x \in M$ and any $\alpha$,

$$
u_{\alpha}(x)=u^{0}(x)+\sum_{i=1}^{N}\left(\frac{\mu_{i, \alpha}}{\mu_{i, \alpha}^{2}+\frac{d_{g}\left(x_{i, \alpha}, x\right)^{2}}{n(n-2)}}\right)^{\frac{n-2}{2}}+R_{\alpha}(x),
$$

where the $R_{\alpha}$ 's, $R_{\alpha} \in H_{1}^{2}(M)$ for all $\alpha$, are such that $R_{\alpha} \rightarrow 0$ strongly in $H_{1}^{2}(M)$ as $\alpha \rightarrow+\infty$. Moreover,

$$
E\left(u_{\alpha}\right)^{2^{\star}}=E\left(u^{0}\right)^{2^{\star}}+N K_{n}^{-n}+r_{\alpha}
$$

for all $\alpha$, where the $r_{\alpha}$ 's, $r_{\alpha} \in \mathbb{R}$, are such that $r_{\alpha} \rightarrow 0$ as $\alpha \rightarrow+\infty$. In other words, the energies split also.

Such a theorem has important applications when dealing with sharp Sobolev inequalities. In this case $N=1$, and the estimate in Theorem 1 appeared to be a key point when discussing the validity and the existence of extremal functions for sharp inequalities like

$$
\|u\|_{2^{\star}}^{2} \leq K_{n}^{2}\|\nabla u\|_{2}^{2}+B\|u\|_{2}^{2}
$$

where $B$ is a constant independent of $u$. Monographs on sharp constant problems are Druet-Hebey [7], and Hebey [12]. Other directions of research are dealing with the energy function (see for instance Hebey [13]) or with compactness results as in Schoen [19, 20. Applications of the theorem in such directions are in Druet [6], where compactness results and low dimension phenomena are discussed, and the lower semicontinuity of the energy function for equations like (2) is proved. The energy function for equations like (2) is defined by

$$
E(\alpha)=\inf _{u \in \mathcal{S}_{\alpha}} E(u)
$$

where $E(u)=\|u\|_{2^{\star}}$ and $\mathcal{S}_{\alpha}$ is the set consisting of the solutions of (2). From the historical viewpoint, without any pretention to exhaustivity, Atkinson-Peletier [1] and Brezis-Peletier [2] have been concerned with the description of the pointwise behaviour of sequences of solutions of equations like (1), dealing with radially symmetrical solutions $u_{\varepsilon}$ of the semicritical equations $\Delta u=u^{2^{\star}-1-\varepsilon}$ on the unit ball of the Euclidean space. More recent developments in this specific direction are in Robert [17, 18. An estimate like in Theorem 11 in the case $N=1$, stating that solutions of minimal energy of equations like (1) are controlled from above by a standard bubble, appeared then in Han [11] when dealing with solutions $u_{\varepsilon}$ of the equations $\Delta u=u^{2^{\star}-1-\varepsilon}$ on bounded open subsets of the Euclidean space, in Hebey-Vaugon [14] when dealing with (11) and arbitrary Riemannian manifolds, and in $\mathrm{Li}[15,16$, when dealing with equations like (11) on the unit sphere. Improvements, still in the case $N=1$, are in Druet [4, 5] and Druet-Robert [10. We refer also to Schoen-Zhang [21]. Examples of blowing-up sequences of solutions of equations like (1) are in Druet-Hebey [8] and Druet-Hebey-Robert [9].

\section{Proof of the theorem}

We only present a very brief sketch of the proof. Details on the special case where $N=1$, which is actually due to Druet and Robert, can be found in the monograph 
[7] by Druet and Hebey. The intricate general case where $N$ is arbitrary is in Druet, Hebey, and Robert [9]. We assume in what follows that $\max u_{\alpha} \rightarrow+\infty$ as $\alpha \rightarrow+\infty$. If not the case, up to a subsequence, the $u_{\alpha}$ 's converge $C^{2}$ to $u^{0}$, and the theorem is true. As a remark, the proof of the theorem goes through the proof of a slightly stronger result. All that follows is up to a subsequence. We let $G$ be the Green's function of the operator $\Delta_{g}+h_{\infty}$, and let $\Phi$ be the continuous function on $M \times M$ given by

$$
\Phi(x, y)=(n-2) \omega_{n-1} d_{g}(x, y)^{n-2} G(x, y)
$$

if $x \neq y$, and $\Phi(x, y)=1$ if $x=y$, where $\omega_{n-1}$ is the volume of the unit $(n-1)$ sphere. Then we claim that for any converging sequence $\left(x_{\alpha}\right)$ of points in $M$, and any $\alpha$,

$$
u_{\alpha}\left(x_{\alpha}\right)=(1+o(1)) u^{0}\left(x_{\alpha}\right)+\sum_{i=1}^{N}\left(\Phi\left(x_{i}, x\right)+o(1)\right) B_{\alpha}^{i}\left(x_{\alpha}\right),
$$

where $x$ is the limit of the $x_{\alpha}$ 's, $x_{i}$ is the limit of the $x_{i, \alpha}$ 's, and $B_{\alpha}^{i}$ is the standard bubble with respect to the $x_{i, \alpha}$ 's and $\mu_{i, \alpha}$ 's given by Theorem 1. It is easily seen that the theorem and the remarks after this theorem follow from such an asymptotic description. The proof of these asymptotics splits into four main steps. The first preliminary step consists in getting rescaling invariant estimates. Such estimates basically state that there exist $N \in \mathbb{N}^{\star}$, converging sequences $\left(x_{i, \alpha}\right)$ in $M$, and sequences $\left(\mu_{i, \alpha}\right)$ of positive real numbers converging to $0, i=1, \ldots, N$, such that

$$
R_{\alpha}^{N}(x)^{\frac{n}{2}-1} u_{\alpha}(x) \leq C
$$

for all $x$ and all $\alpha$, where $R_{\alpha}^{N}(x)$ is the minimum over $i$ of the distances from the $x_{i, \alpha}$ 's to $x$. The key idea here is that if such an estimate is false, then we can construct another blow-up point. This weak estimate comes with an important refinement and complementary informations on the limit of the $u_{\alpha}$ 's outside blowup points. Then we need to prove that the upper estimate in Theorem 1 holds. For that purpose, we rearrange the $x_{i, \alpha}$ 's in families. Inside a family, blow-up points are close to each other. Two families are far from each other. If the $y_{i, \alpha}$ 's are the representatives of such families having the largest $\mu_{i, \alpha}, i=1, \ldots, k$, the second step in the proof consists in proving that an upper estimate like in Theorem 1 holds with respect to the $y_{i, \alpha}$ 's. We prove that there exist $C>0$ and $R>0$ such that

$$
u_{\alpha}(x) \leq(1+o(1)) u^{0}(x)+C \sum_{i=1}^{k} B_{\alpha}^{i}(x)
$$

for all $\alpha$ and all $x \in M \backslash \bigcup_{i=1}^{k} B_{y_{i, \alpha}}\left(R \mu_{i, \alpha}\right)$, where $B_{\alpha}^{i}$ is the standard bubble with respect to $y_{i, \alpha}$ and its corresponding $\mu_{i, \alpha}$. The proof of such an estimate goes through the establishment of a scale of intermediate estimates, referred to as $\varepsilon$ sharp estimates with $0<\varepsilon<\frac{n-2}{2}$, the weakest of these when $\varepsilon=\frac{n-2}{2}$ being like the weak estimate we discussed above. The proof of the upper estimate as in the theorem then reduces to the proof that this estimate holds inside the $B_{y_{i, \alpha}}\left(R \mu_{i, \alpha}\right)$ 's. This is the third step in the proof. We proceed here by induction. We consider subfamilies of blow-up points, and prove that the estimate holds in $B_{y_{i, \alpha}}\left(R \mu_{i, \alpha}\right)$, outside smaller balls of sub-representatives, and so on up to the point where we have exhausted all the blow-up points. In each step of this induction process, we pass through $\varepsilon$-sharp estimates, and, in some sense, let then $\varepsilon \rightarrow 0$ to get the sharp 
estimate. Once we have proved that the upper estimate of Theorem 1 holds in $M$, the argument becomes simpler and more conventional. The fourth step in the proof consists in proving that the above asymptotics follow from the Green representation formula

$$
\begin{aligned}
u_{\alpha}\left(x_{\alpha}\right)-u^{0}\left(x_{\alpha}\right)= & \int_{M} G_{\alpha}\left(x_{\alpha}, x\right)\left(u_{\alpha}(x)^{2^{\star}-1}-u^{0}(x)^{2^{\star}-1}\right) d v_{g} \\
& +\int_{M} G_{\alpha}\left(x_{\alpha}, x\right)\left(h_{\infty}(x)-h_{\alpha}(x)\right) u^{0}(x) d v_{g}
\end{aligned}
$$

where $G_{\alpha}$ is the Green's function for the operator $\Delta_{g}+h_{\alpha}$. The different terms that are involved in this formula are controlled thanks to the upper estimate we have just discussed. The asymptotics follow from rather standard developments. As already mentioned, since $\Phi$ is continuous, the theorem and the remarks after the theorem are then easy consequences of the asymptotics. We refer to Druet, Hebey and Robert [9] for more details, and also to Druet and Hebey 7] for the special case where $N=1$.

\section{REFERENCES}

1. Atkinson, F.V., and Peletier, L.A., Elliptic equations with nearly critical growth, J. Diff. Equ., 70, 349-365, 1987. MR 89e:35054

2. Brézis, H., and Peletier, L.A., Asymptotics for elliptic equations involving critical Sobolev exponents, in Partial Differential Equations and the Calculus of Variations, eds. F. Colombini, A. Marino, L. Modica and S. Spagnalo, Basel: Birkhaüser, 1989. MR 91a:35030

3. Caffarelli, L. A., Gidas, B., and Spruck, J., Asymptotic symmetry and local behavior of semilinear elliptic equations with critical Sobolev growth, Comm. Pure Appl. Math., 42, 271-297, 1989. MR 90c:35075

4. Druet, O., The best constants problem in Sobolev inequalities, Math. Ann., 314, 327-346, 1999. MR 2000d:58033

5. Sharp local isoperimetric inequalities involving the scalar curvature, Proc. Amer. Math. Soc., 130, 2351-2361, 2002. MR 2003b:53036

6. - From one bubble to several bubbles. The low-dimensional case, Preprint, 2002.

7. Druet, O., and Hebey, E., The AB program in geometric analysis. Sharp Sobolev inequalities and related problems, Memoirs of the American Mathematical Society, MEMO/160/761, 2002.

8. Druet, O., and Hebey, E., Blow-up examples for second order elliptic PDEs of critical Sobolev growth, Preprint, 2002.

9. Druet, O., Hebey, E., and Robert, F., Blow-up theory for elliptic PDEs in Riemannian geometry, Preprint, 201 pages, 2002.

10. Druet, O., and Robert, F., Asymptotic profile for the sub-extremals of the sharp Sobolev inequality on the sphere, Comm. P.D.E., 25, 743-778, 2001. MR 2002k:58045

11. Han, Z.C., Asymptotic approach to singular solutions for nonlinear elliptic equations involving critical Sobolev exponent, Ann. Inst. H. Poincaré. Anal. Non Linéaire, 8, 159-174, 1991. MR 92c:35047

12. Hebey, E., Nonlinear analysis on manifolds: Sobolev spaces and inequalities, CIMS Lecture Notes, Courant Institute of Mathematical Sciences, Vol. 5, 1999. Second edition published by the American Mathematical Society, 2000. MR 2000e:58011

13. - Nonlinear elliptic equations of critical Sobolev growth from a dynamical viewpoint, Preprint, Conference in honor of H. Brézis and F. Browder, Rutgers university, 2001.

14. Hebey, E., and Vaugon, M., The best constant problem in the Sobolev embedding theorem for complete Riemannian manifolds, Duke Math. J., 79, 235-279, 1995. MR 96c:53057

15. Li, Y.Y., Prescribing scalar curvature on $S^{n}$ and related problems, Part I, J. Diff. Equations, 120, 319-410, 1995. MR 98b:53031

16. _ Prescribing scalar curvature on $S^{n}$ and related problems, Part II: existence and compactness, Comm. Pure Appl. Math., 49, 541-597, 1996. MR 98f:53036

17. Robert, F., Asymptotic behaviour of a nonlinear elliptic equation with critical Sobolev exponent-The radial case I, Adv. Differential Equations, 6, 821-846, 2001. MR 2002d:35054 
18. _ Asymptotic behaviour of a nonlinear elliptic equation with critical Sobolev exponentThe radial case II, Nonlinear Differ. Equ. Appl., 9, 361-384, 2002.

19. Schoen, R., Variational theory for the total scalar curvature functional for Riemannian metrics and related topics, Topics in calculus of variations (Montecatini Terme, 1987), 120-154. Lecture Notes in Mathematics, 1365, Springer, Berlin-New York, 1989. MR 90g:58023

20. - On the number of constant scalar curvature metrics in a conformal class, Differential Geometry: A symposium in honor of Manfredo Do Carmo, H.B.Lawson and K.Tenenblat eds., Pitman Monogr. Surveys Pure Appl. Math., 52, 311-320, 1991. MR 94e:53035

21. Schoen, R., and Zhang, D.,Prescribed scalar curvature on the $n$-sphere, Calc. Var. Partial Differential Equations, 4, 1-25, 1996. MR 97j:58027

22. Struwe, M., A global compactness result for elliptic boundary problems involving limiting nonlinearities, Math. Z., 187, 511-517, 1984. MR 86k:35046

Département de Mathématiques, Ecole Normale Supérieure de Lyon, 46 allée d’Italie, 69364 Lyon CEDEX 07, France

E-mail address: Olivier.Druet@umpa.ens-lyon.fr

Département de Mathématiques, Université de Cergy-Pontoise, 2 avenue Adolphe Chauvin, 95302 Cergy-Pontoise Cedex, France

E-mail address: Emmanuel.Hebey@math.u-cergy.fr

Department of Mathematics, ETH Zürich, CH-8092 Zürich, Switzerland

E-mail address: Frederic.Robert@math.ethz.ch 